\title{
CONDITION-BASED MAINTENANCE MODEL FOR THE OPTIMIZATION OF SMART MANUFACTURING PROCESSES
}

\author{
C. C. Rusul*, S. Belaid², L. R. Mistodiel, S. Adjerid² \\ 1"Dunarea de Jos" University of Galati, Faculty of Engineering, Department of Manufacturing Engineering, \\ 111 Domneasca St., 800201 - Galati, Romania \\ ${ }^{2}$ Université M'Hamed Bougara de Boumerdes, Faculty of Engineering Sciences, Department of Mechanical \\ Engineering, Cite Frantz. Fanon, 35000 - Boumerdes, Algerie \\ *Corresponding author's e-mail address: carmen.rusu@ugal.ro
}

\begin{abstract}
The research focused on the elaboration of the Condition-Based Maintenance (CBM) for the optimization of Smart Manufacturing Processes (SMP) using three types of condition monitoring (CM). The paper presents some considerations regarding the Smart Manufacturing Processes (SMP), especially focused on the Additive Manufacturing (AM) process, by Fused Deposition Modelling (FDM) technology and the way in which the CM and mathematical and probabilistic models can be used, for achieving CBM. The experiments focused on testing three different types of CM: vibration (by vibrometers), sound (by sound level meters) and temperature (by thermal camera and infrared thermography). The experiments and results were analysed, data were processed and a probabilistic model was designed, based on statistical methods, using features/key indicators and their thresholds values. Furthermore, a specific model based on Bayesian network was developed, for achieving CBM of the SMP, specifically to the AM/FDM process. This technique and approach can represent a successful integration of a large number of data monitoring sets and complex modelling and analysis capabilities which can lead in the end to an optimisation of the SMP.
\end{abstract}

KEYWORDS: Condition-Based Maintenance (CBM), Condition Monitoring (CM), Additive Manufacturing (AM), Fused Deposition Modelling (FDM), Infrared Thermography (IRT), vibration analysis, sound analysis, Bayesian networks (BNs).

\section{INTRODUCTION}

The smart manufacturing industry is continuously increasing its efficiency and effectiveness by improving the supply chains, for meeting the requirements in terms of productivity and performance [1], [2]. Furthermore, the concept of smart manufacturing processes (SMP) involves systems capabilities to be flexible, robust, and adaptive. However, the introduction of lean manufacturing increases issues of equipment availability, and therefore, an effective maintenance is compulsory to industry sector [3], [4], [5].

One of the cutting-edge technologies that is considered a SMP is the additive manufacturing (AM), also known as 3D printing. The definition provided by the International Standardisation Organisation and American Society for Testing and Materials (ASTM) in the unified standard ISO/ASTM 52900:2015 is that $\mathrm{AM}$ is "the general term for those technologies that, based on a geometrical representation, create physical objects by successive addition of material". The standard also highlights various domains of applications in engineering industry, and also medicine, education, architecture, and creative industries [6]. The SMP challenges in AM technologies are related to fostering the design for user-friendly, safe and self-contained, flexible and integrate systems that could provide rapid prototyping, reverse engineering methods and speed, doubled by quality, resolution and no further additional finishing of the 3D objects [7], [8].

For these reasons, the maintenance is becoming very important for the manufacturing industry, due to the worldwide industrialization agendas, that includes the concepts of Industry 4.0, industrial internet of things (IIoT), cyber-physical systems (CPS), including artificial intelligence (AI), machine learning (ML) technologies, in a digital transformation era. These technologies will promote the collaborative connection between equipment, devices and enterprise assets, using Internet, Big Data solutions and Cloud Computing [9], [10], [11]. In terms of maintenance, the concept refers to the elaboration of a policy that should 
be applied in agreement with the manufacturing company's management, that should take into consideration the objectives, but also the operations, materials proprieties, the behaviour of the equipment in the production flow, the application conditions, and the costs. For this reason, in the manufacturing industry there are two main types of maintenance used: corrective maintenance and preventive maintenance [12]. These types of maintenance, applied in the manufacturing environments, require a detailed optimisation of the production planning and control in order to avoid a potential slow down or shut down of the production lines, poor operational activities, potential risk of injury of the workers, flaws in the raw material flow, which will increase the cost of work-inprocess. Proper planning should include cost-based, availability-based and reliability-based approaches, in order to proper schedule the number of interventions and the optimum maintenance interval [13], [14], [15].

Condition-Based Maintenance (CBM) is an industrial program that puts forward strategic maintenance actions upon data acquired through Condition Monitoring (CM), that is related to the acquisition and processing of information and data that indicate the state of a machine over time [8]. CBM includes integrated CM methods, in order to avoid unnecessary intervention actions and decides when the maintenance is required in cases of abnormal behaviours of the systems, equipment or devices [16], [17]. A strategic CBM plan accurately implemented can significantly reduce intervention costs by reducing the number of unnecessary scheduled preventive or corrective maintenance operations [3], [4], [18].

The CBM is related to three important issues:

- Detection - when the fault is developing, at an early stage;

- Diagnosis - what is the nature of a fault and the examination;

- $\quad$ Prognosis - forecasting the trend and enabling the maintenance plan.

In order to attain the forecasting process for $\mathrm{CMB}$, there are various CM technologies that can be used for diagnosis and analysis of the data acquired from the manufacturing processes flow: vibration, ultrasonic, oil and noise analysis and, also, infrared thermography. In figure 1 are presented the main CM methods their threshold levels which forecast the maintenance time and predicts the breakdown of the systems, equipment or devices [19].

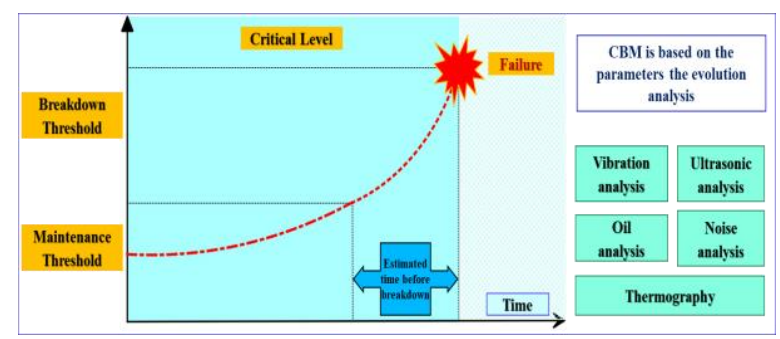

Fig. 1. CM methods and threshold levels for CBM [19]
There are many applications of the SMP, where the sensors get significantly bent, and undergo changes in temperature and humidity, which could be detrimental to the reliability of data being collected. This requires a strategic plan of the measurements in order to gather reliable and relevant data. This plan should include adjustments and calibrations of the devices according to the specific standards, testing of the measurement systems in different environmental conditions, and taking into consideration the lurking variables. This plan helps researchers in the identification of methods for reducing variations and minimising the data inconsistency, and achieving experimental accuracy.

As a conclusion, based on the researches in the scientific literature, it can be said that the CBM is a complex fundamental and experimental field, that uses advanced devices and equipment for processes monitoring, statistical analysis and high-level algorithms for data processing, in order to identify the specific times for performing the maintenance process. Taking into consideration all the above, the authors developed a system that included different devices. The main goal of this research is to investigate the condition monitoring techniques in the smart monitoring processes, specifically where advanced manufacturing technologies can be applied.

\section{EXPERIMENTAL PART}

The experiments were conducted in the laboratory of Mechatronic Systems from "Dunarea de Jos" University of Galati, Faculty of Engineering, Manufacturing Engineering Department. This included the analysis of the CBM based on infrared thermography, vibration analysis and acoustics, on the AM process achieved by using the Creality CR-10S PRO 3D printer [20]. The 3D printer is using the fused deposition modelling (FDM) technology, with automatic and auxiliary levelling modes, filament detection (PLA, ABS, wood, TPU, carbon fibre), double gear extrusion mechanism, a motherboard with four-layer PCB, TMC ultra-quiet drive 256 subdivision, for precision printing. The printing size is $300 \times 300 \times 400 \mathrm{~mm}$ and can achieve up to $60 \mathrm{~mm} / \mathrm{s}$ printing speed [21]. It is using both Cura, and Simplify $3 D$ slicing software. The main scope of the research was to analyse three different methods of conditionbased monitoring for achieving CBM, using three techniques, by measuring: the temperature (by thermal camera and IRT), the accelerations/vibrations (by vibration meters), and the sound (by a sound meter).

The design of the research methodology (Fig. 2) included the setting on a framework with devices, software and techniques for achieving the CM. The positioning of the devices can be observed as well as the connections between these and the main methods of CM in the real conditions of the AM-FDM process. In the experiments, the piece P1, a clamping system (Fig. 3), was printed using the Creality CR-10S Pro 3D. 


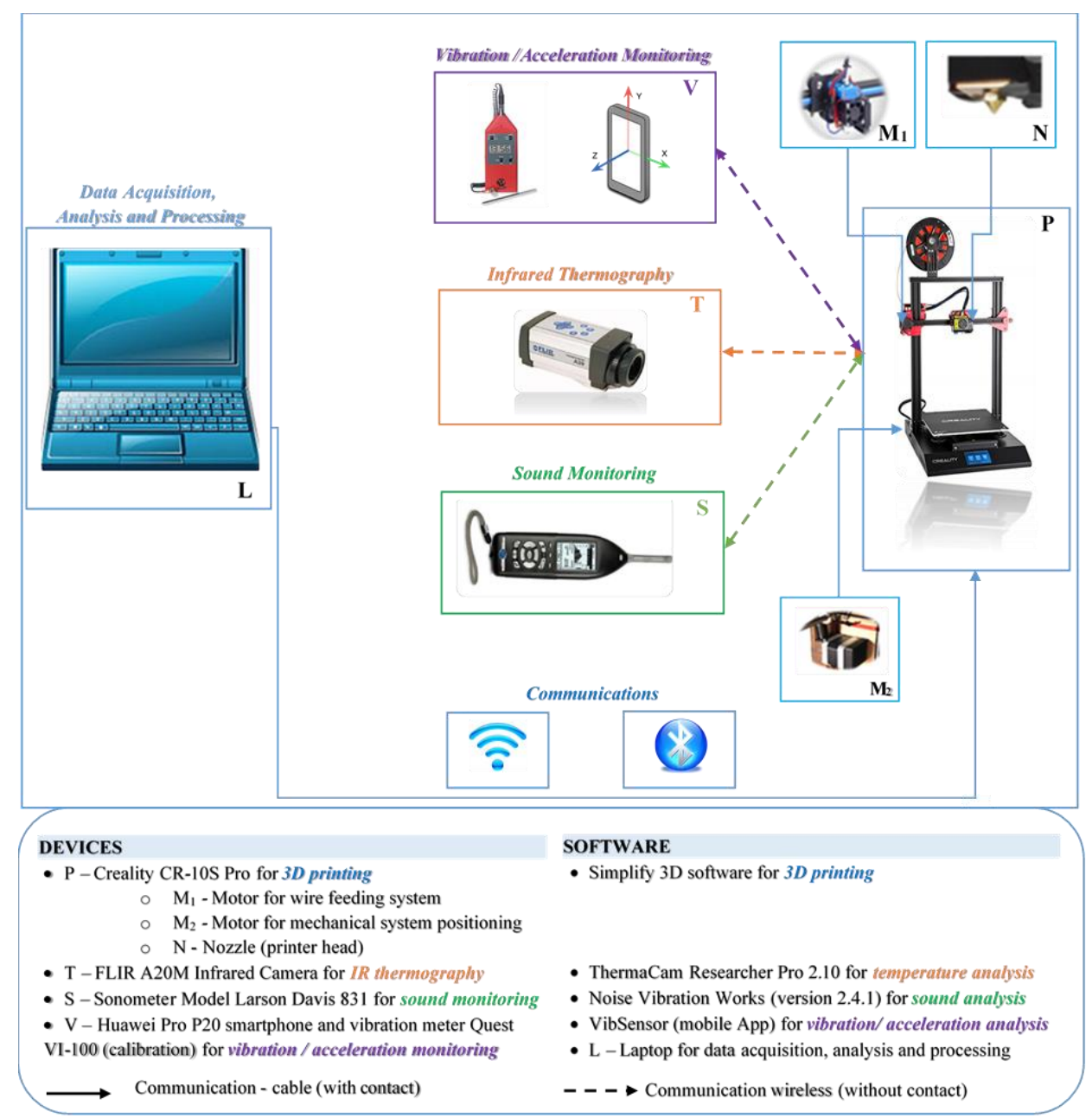

Fig. 2. Research methodology

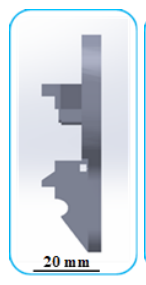

a)

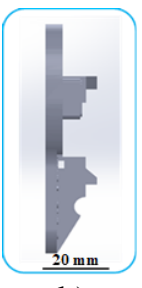

b)

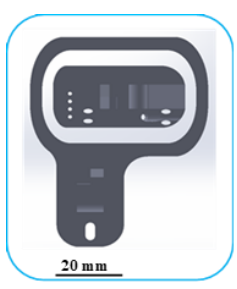

c)

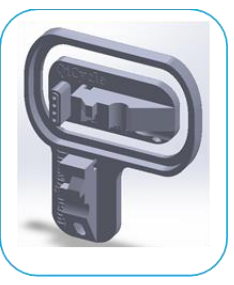

d)
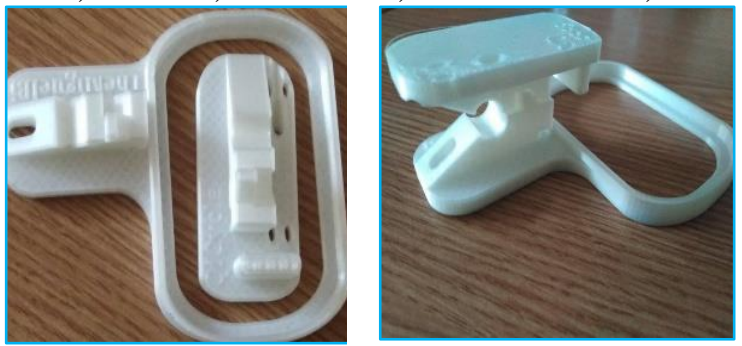

f)

Fig. 3. The 3D model of the piece P1 (a clamping system); left a), right b), front c), isometric d) and final printed parts e-f

The AM-FDM process that took place about $3 \mathrm{~h}$ and 50 minutes. The 3D model was created in SolidWorks and exported like STL file. Afterwards, using the Simplify $3 D$ software was created the G-code file, and imported

in the 3D printer flash-drive. The material for printing the piece was polylactic acid (PLA) with the printing temperature between $180-230^{\circ} \mathrm{C}$ and build platform temperature of $20-60^{\circ} \mathrm{C}$. The experiments followed the $\mathrm{CM}$ of the Creality 10S Pro 3D printer nozzle area and two motors, respectively NEMA 17 stepper motors [22]. The stepper motors are DC motors with multiple coils, allowing them to move in small increments. These are used for the printer head positioning, speed control, and low speed torque, and also for the printer wire feeding system. These motors are very accurate and feasible still, they have their limitations. At start, the stepper motor current consumption is independent of load. When a motor is not moving, it will still consume a large amount of current. Stepper motors also tend to lose torque at high speeds. Some stepper motors are designed for high speed torque, but the performance of these motors is also greatly dependent on the stepper drivers paired with them. Thereby, the $\mathrm{CM}$ of the 3D printer motors is crucially for achieving high quality $3 \mathrm{D}$ parts and proper functionality of the printer. Furthermore, for analysing the behaviour of the motors and of the 3D printer nozzle, during the AMFDM process, the research experimental design followed is presented din figure 2 . 


\subsection{IRT Monitoring}

In the field of CBM, infrared thermography is a widely used technique for evaluating part conditions and is a very important tool for ensuring the safety and reliability of the equipment. For the IRT monitoring, in the experiments, the ThermoVision A20M thermo camera (Fig. 4) was used, a device that allows to measure temperature differences as small as $0.12^{\circ} \mathrm{C}$ in a range from $-20^{\circ} \mathrm{C}$ up to $+900^{\circ} \mathrm{C}$. It produces highresolution images (320 x 240 pixels), offering more than 76,800 individual measurement points per image at a refresh rate of $50 / 60 \mathrm{~Hz}$. The data can then be used by the operators to monitor or control the production processes, or can be processed by the camera's onboard intelligence to autonomously generate multiple independent digital alarms or even control process equipment [23]. The IRT is appointed by the method potential and efficiency, that allows to detect temperature variation, revealing the appearance of major defects that may affect the operational safety of the production equipment. The positioning of the camera wat at 1-meter distance from the monitored area and the specific parameters of emission, ambient temperature and humidity were introduced for the condition monitoring.

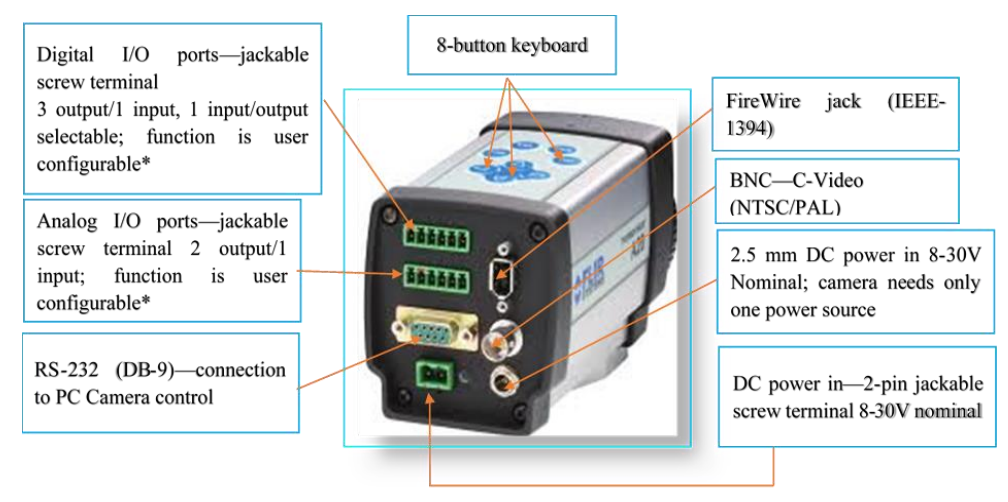

Fig. 4. ThermoVision ${ }^{\mathrm{TM}}$ A20M camera [20]

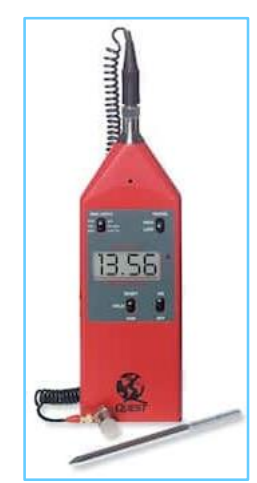

Fig. 5. Vibrometer Quest VI-100 [24]

\subsection{Vibration Monitoring}

The second part of the experiments was related to the vibration encountered during the AM-FDM process. For the vibration monitoring, two methods of analysis were used: a contact condition monitoring, using a smart phone that was positioned on the $3 \mathrm{D}$ printer during the process, and an industrial device for the calibration of the measurements. The measurements were performed using a Huawei P20 smart phone (Fig. 6a) that included the accelerometer sensors, and measures along each of the main axes of the device. By convention, the axes are labelled as figure $6 \mathrm{~b}$ presents. On the device, a mobile application called VibSensor was downloaded from Google Play and installed for recording data (Fig. 6c).
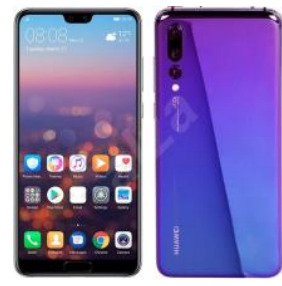

a)

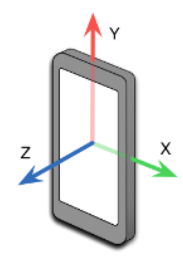

b)

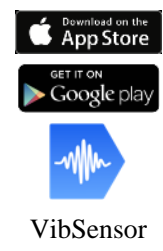

c)
Fig. 6. Device for real time vibration monitoring

The industrial vibrometer Quest VI-100 was used for calibration, and it is a device that can evaluate equipment vibrations and estimate the effectiveness of isolation. The VI-100 is suitable for general-purpose industrial or environmental applications. It measures shock or pulsation, basic machinery condition monitoring, makes comparative studies, quality specification checks and can be used in calibration of different other vibrometers [24]. The vibration range that Quest VI-100 can measure for acceleration is between 0.01 and $199.9 \mathrm{~g}$, for displacement between 0.01 to $199.9 \mathrm{~m} \mathrm{x} 10^{-5}$ and velocity between 0.01 to $199.9 \mathrm{~cm} / \mathrm{sec}$. The supported frequency range is between 5 to $10,000 \mathrm{~Hz}$ with analog output in $\mathrm{AC}$ signal of maximum $5 \mathrm{~V}$, relative to signal ground, and in $\mathrm{DC}$ for high range a signal of maximum $2 \mathrm{~V}$ relative to signal ground, and $10 \mathrm{mV}$ of root mean square (RMS) signal per count of 1.0 in the display and for low range $100 \mathrm{mV}$ of RMS signal per count of 1.0 in the display.

\subsection{Sound Monitoring}

The third part of the experiments was related to the sounds encountered during the AM-FDM process. In the field of non-destructive testing, acoustic emission is a method that allows to perceive in real time the occurrence of a signal resulting from a cohesion in a material. It is also a method of voluminal control due to the emission and propagation of elastic waves. The measurements were performed using a Larson Davis 831 Sonometer model [25].

The Larson Davis Model 831 Class 1 Sound Level Meter, with its high definition display, is a versatile device, performing the functions of several 
instruments. It combines the features of a precision Class 1 sound level meter, environmental noise analyser, personal noise dosimeter, and a real-time frequency analyser. The Model 831 is a fifth generation Larson Davis sound level meter, designed for simple, single-handed operation, is fully featured, smart and versatile with an ever-expanding firmware platform. The design of the Model 831 was based on countless inputs from customers. It expands upon the Larson Davis tradition of delivering value, innovation and function in a rugged, single-handed, expandable package and is backed by a 2-year factory warranty, 24-hour application support and accredited factory service/calibration [22].

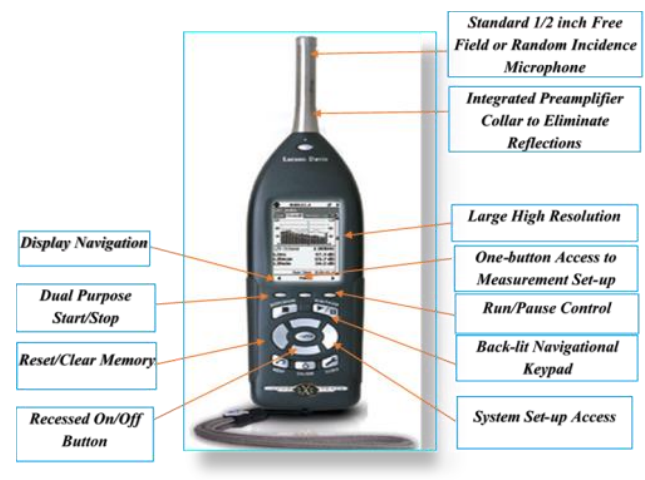

Fig. 7. Sound Meter Larson Davis 831 [22]

\subsection{Data Analysis and Processing Software}

In the experiments, several programs have been used for the design, analysis and monitoring of the smart manufacturing process, in this case the $3 \mathrm{D}$ printing process:

1. ThermaCAM Researcher ${ }^{\mathrm{TM}}$ from Flir: monitors and analyses changes and temperature variation in both motors and nozzle area. It digitally stores and retrieves static and real-time infrared images, digital video sequences, dynamic high-speed events and data directly, allowing in-depth and analysis of thermal events [26].

2. Noise \& Vibration Works (NWWin): analyse the results of vibratory and acoustic monitoring. NWWin is a software designed specifically for handling Noise
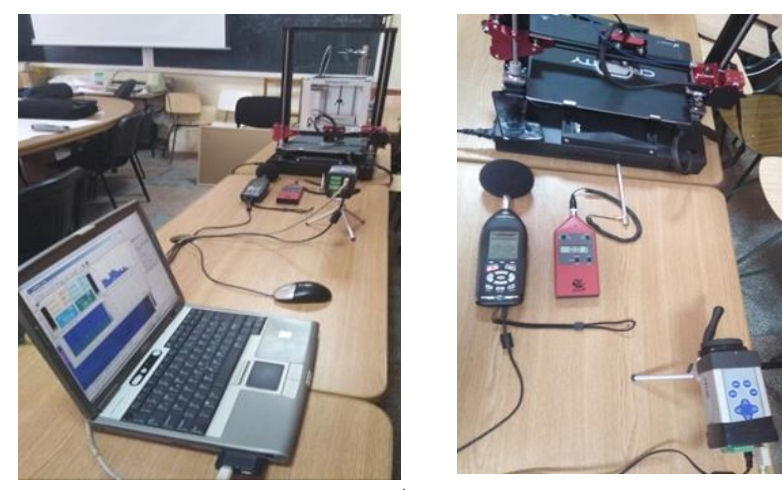

a) and Vibration data, that provides reporting and application modules for Environmental, Workplace, Sound Power, Architectural, and Automotive Pass-by. It can handle the native files from all Larson-Davis and Sinus instruments as well as any meter that can export *.csv format [27].

3. VibSensor is a mobile application for vibration recording, analysis and data storage. The application is used for live displaying of the acceleration, speed and displacement data in real time. The acquired data are compared to the data retrieved from the industrial vibration measurement device VI-100 [28].

4. Cura for the $3 D$ printing: creates the integration between compatible 3D printers, software and materials and it is an open source cross-platform. It has plugins for any CAD software and optimized profiles for third-party materials and supports STL, OBJ, X3D, and 3MF file formats [29].

5. Simplify $3 D$ is a software that includes a realistic pre-print simulation, allowing to see the exact actions of the 3D printers, that will perform before starting the printing. The simulation includes information about the exact speeds, sequences, and printer settings, so can quickly verify the setup from the beginning [30].

\section{RESULTS AND DISCUSSION}

\subsection{Temperature Field Distribution}

The IRT monitoring was performed during the entire period of the AM-FDM process (Fig. 8). This included the $\mathrm{Z1}$ phase, where the printer calibration was achieved and included the period of printer head heating, with a total time of 8 minutes, the $\mathrm{Z} 2$ phase in which the AM-FDM process was carried out, and lasted for 177 minutes, and the Z3 cooling phase, that started from the minute 185 , as figure 9 to figure 11 show. Three points from the motor $\mathbf{M}_{1}$ were chosen for analysis and used for the wire feeding system, respectively the spots SP1, SP2 and SP3 and three points from the motor $\mathrm{M}_{2}$ used for the mechanical system positioning, respectively the spots SP4, SP5 and SP6, positioned on the axial line of the motors. Moreover, the zone from the 3D printer nozzle (printer head) was marked using an area, AR1 respectively, in order to determine the maximum temperature.

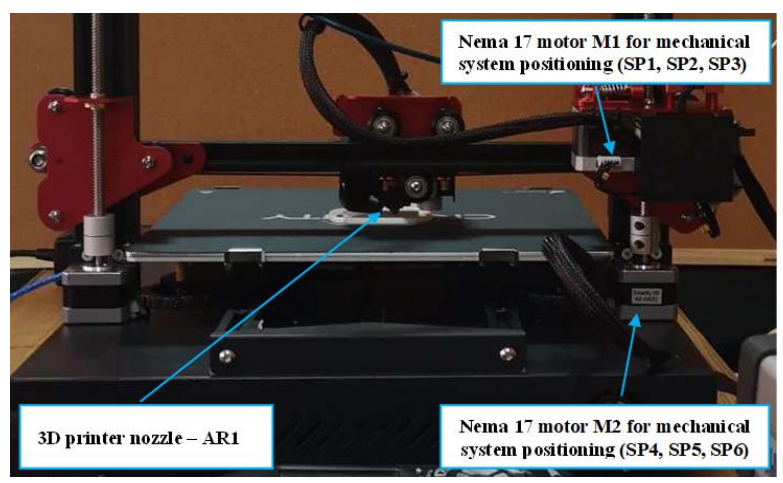

b)

Fig. 8. AM process monitoring using IRT: a) experimental setup; b) monitored areas during the process 


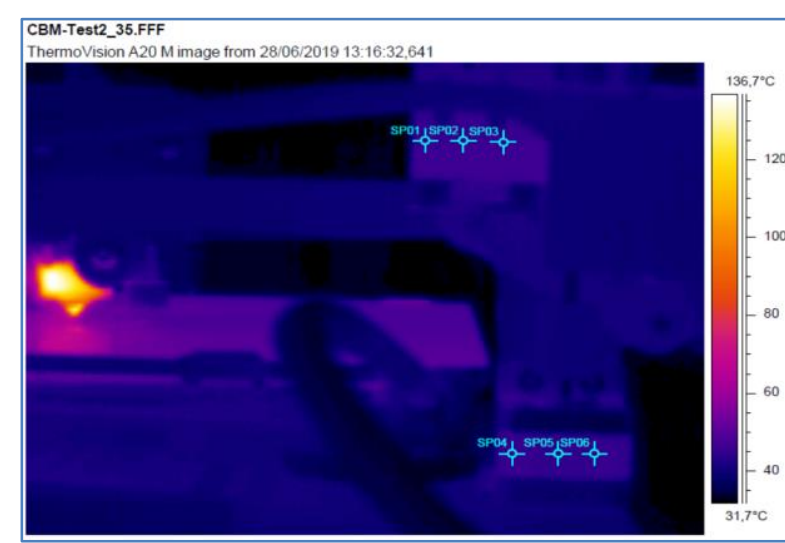

Fig. 9. Temperature field distribution at $\min 35$, when SP01-6 from the $M_{1}$ and $M_{2}$ reached the maximum temperature

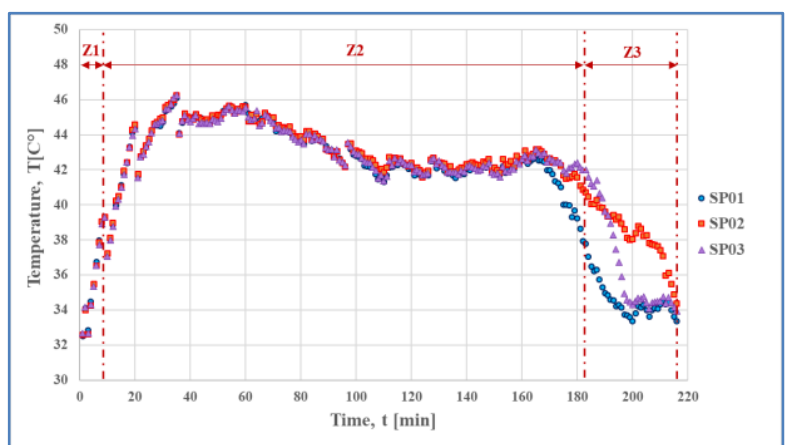

a)

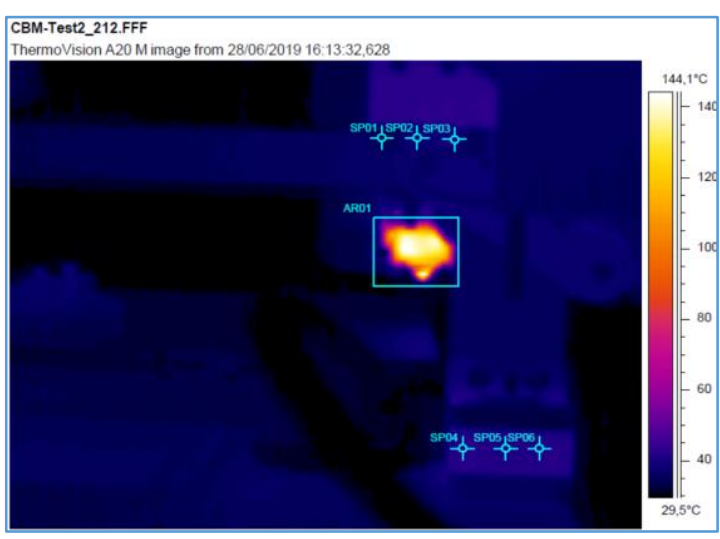

Fig. 10. Temperature field distribution in the nozzle area AR01, when maximum temperature was reached, at the end of the $3 \mathrm{D}$ printing

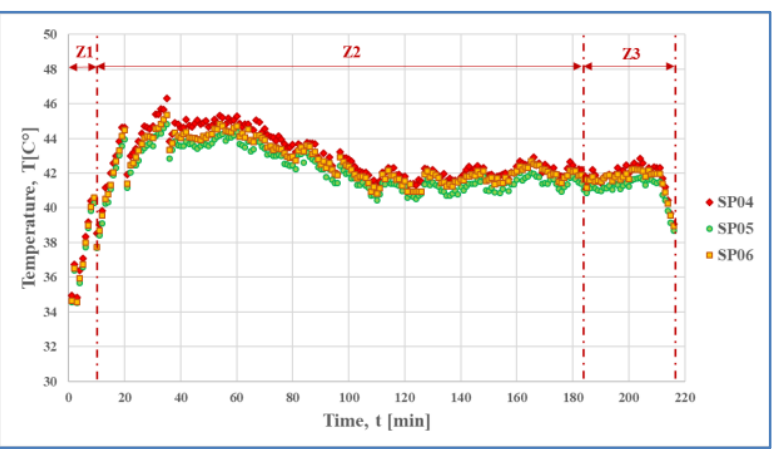

b)

Fig. 11. Temperature variation during the AM-FDM process: a) on the motor $M_{1}$ in SP1, SP2 and SP3; b) on the motor $\mathrm{M}_{2}$ in SP4, SP5 and SP6

In figure 11 is shown the temperature field distribution during the AM-FDM process, when the maximum temperature on the motor $\mathrm{M}_{1}$ and motor $\mathrm{M}_{2}$ was achieved. Moreover, as figure 11a and $\mathrm{b}$ show, the maximum temperature reached during the entire period of the AM-FDM process - $\mathrm{Z} 2$ in the $\mathrm{M}_{1}$ was achieved at time $\mathrm{t}=35 \mathrm{~min}$, in $\mathrm{SP} 01$ at a temperature of $46.109^{\circ} \mathrm{C}$, SP02 and SP3 of $46.25^{\circ} \mathrm{C}$. As predicted, the maximum temperature in motor $\mathrm{M}_{2}$ was achieved at the same time $\mathrm{t}=35 \mathrm{~min}$, in SP04 at a temperature of $46.31^{\circ} \mathrm{C}, \mathrm{SP} 05$ of $44.83^{\circ} \mathrm{C}$ and SP06 of $45.35^{\circ} \mathrm{C}$.

The minimum temperature from the $\mathrm{Z} 2$ was achieved at the end of the AM-FDM process, at $\mathrm{t}=185 \mathrm{~min}$, respectively on the motor $\mathrm{M}_{1}$ of $36.49^{\circ} \mathrm{C}$ in SP01 of $40.06^{\circ} \mathrm{C}$ in SP02 and of $41.21^{\circ} \mathrm{C}$ in Sp03, and on the motor $\mathrm{M}_{2}$ of $41.06^{\circ} \mathrm{C}$ in $\mathrm{SP} 04$, of $40.44^{\circ} \mathrm{C}$ in SP05 and of $40.82^{\circ} \mathrm{C}$ in SP06.

It can be also noticed that the temperature variation of the points SP01-06, for both motors, follows a similar trendline during the AM-FDM process. However, an inflection point in the $\mathrm{Z} 2$ area occurred at time $\mathrm{t}=110 \mathrm{~s}$, where the temperature of the motors decreased as in $\mathrm{M}_{1}$ in SP01 at $41.32^{\circ} \mathrm{C}$, in SP02 at $41.86^{\circ} \mathrm{C}$ and in $\mathrm{Sp} 03$ at $41.36^{\circ} \mathrm{C}$, as well as the $\mathrm{M}_{2}$ in SP04 at $41.46^{\circ} \mathrm{C}$, in $\mathrm{Sp} 05$ at $40.72^{\circ} \mathrm{C}$, and in SP06 at $40.95^{\circ} \mathrm{C}$. This phenomenon is explained by the fact that the printer finished printing the outer (exterior) part of the piece and after time $\mathrm{t}=110 \mathrm{~min}$ it started printing the inner part (interior) as it can be seen in figure $3 \mathrm{c}$.

Because of the fact that the area chosen to represent the temperature measurements for the nozzle zone, respectively AR01 is fixed, and because of the fact that, in real time condition of the AM-FDM process, the nozzle is mobile, moving along the $3 \mathrm{D}$ printer plate in order to print the $3 \mathrm{D}$ object, it is very difficult to achieve data processing of the specific area. For this reason, other methods of condition monitoring can be applied for the nozzle area, and other solutions should be investigated in order to obtain proper IRT results and temperature thresholds.

\subsection{Vibration Recording and Analysis}

The second CM applied on the AM-FDM process is the vibration monitoring, analysis and data processing. This was achieved by using the VibSensor mobile app, that records, analyses, stores, and emails accelerometer and vibration data, offering live display of the tilt and vibration data in real time, acquisition (timed acquisition, with settable delay and duration; it collects raw accelerometer data for up to 10 minutes at max rate allowed by device), data storage (on the device, with date and time stamp for later retrieval, analysis (data collection can be viewed to see the raw accelerometer data, processed tilt and vibration, and calculated power spectral densities, both in graphical and report format) 
and email access features, in which users can email raw or processed data in text (csv or tab-delimited) or MATLAB format. The measurements were performed on the entire AM-FDM process $\mathrm{Z} 2$, in the same time with the IRT [28]. The raw accelerometer data contains the effects of gravity plus any other accelerations the device may be experiencing. In Live View, this data is split into slowly varying Tilt and quickly varying Vibration data. For tilt data, a full hemisphere means one $g$ of acceleration. The scale for vibration data is indicated by the small-scale bar, and ranges from 0 to $0.5 \mathrm{~g}$. Typical devices actually have a full range of $-2 \mathrm{~g}$ to $+2 \mathrm{~g}$. The smaller range in Live View is chosen to emphasize smaller vibrations. The data acquisition related to the vibrations of the $3 \mathrm{D}$ printer was triggered at the beginning of the manufacturing process. Each data consisted of raw, time-stamped acceleration information, that was automatically saved into a database, with a date and time stamp that allows later viewing, analysing, and exporting in different formats, such as *.csv, *.txt or *.m files. The frequency range of acquisition can be modified in the settings. The "high" range collects at the maximum rate supported by the device and allows analysing vibrational frequencies between 0.03 and $50 \mathrm{~Hz}$. The "low" range extends this by averaging to allow analysis of vibrations with periods as long as 5 minutes [28].

In figure 12 is presented the power spectral density (PSD) in the case of the vibration testing, or better said the acceleration spectral density measured and logged using the Huawei P20 device and VibSensor mobile application. The PSD allows to interpret which are the strongest and weakest vibration frequencies. Furthermore, the application gives information related to the following data:

-Length: 229 min $57 \mathrm{sec}$

-Points: 46.173

-Gaps: 10 from 13377 sec total

-Data rate: $100.0 \mathrm{~Hz} \quad$ Units: $\mathrm{m} / \mathrm{s}^{2}$

-Peak raw: X (Limit) Y (11.64) Z (Limit)

-Integrated Spectral Density - ISD:

X (0.28) Y (0.41) Z (0.28)

-Resonances:

X: $0.13 \mathrm{~Hz}(0.078), 4.5 \mathrm{~Hz}(0.0068)$

Y: $0.085 \mathrm{~Hz}(0.59), 7.4 \mathrm{~Hz}(0.021)$

Z: $0.024 \mathrm{~Hz}(0.033), 32 \mathrm{~Hz}(0.026)$

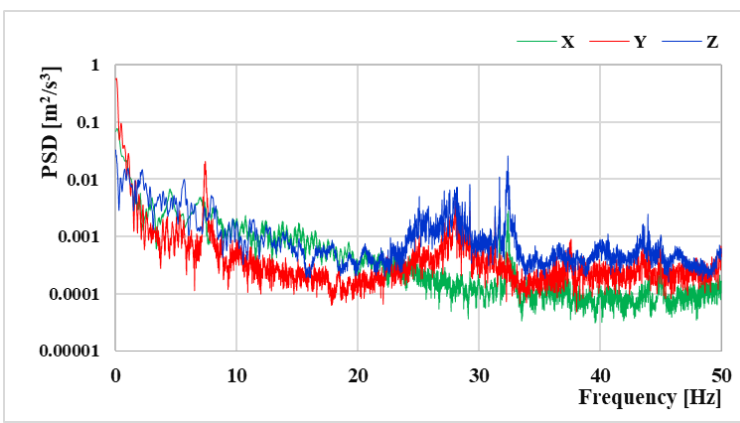

Fig. 12. Vibration Power Spectral Density measured using smartphone and VibSensor application
The resulted data were compared to the Vibration meter Quest model VI-100 industrial vibration meter in order to calibrate the sensors and also to validate the measurements. VibSensor application installed on the Huawei P20 smartphone has proven to be reliable and accurate for the proposed measurements for monitoring the $3 \mathrm{D}$ printing process.

\subsection{Sound Recording and Analysis}

The third part of experiments was related to the sound encountered during the $3 \mathrm{D}$ printing process. In the field of non-destructive testing, acoustic emission is a method that allows to perceive in real time the appearance of a signal resulting from a cohesion in a material. It is also a method of voluminal control due to the emission and propagation of elastic waves. The measurements were performed using a Larson Davis 831 Sonometer model [25]. Figure 13 shows the measured and logged sound signals during the AMFDM processes. The data processing and analysis were performed using NWWin and the main parameters measured were the $\mathrm{A}$ and $\mathrm{C}$ weighted equivalent continuous sound levels in decibels $-\mathrm{L}_{\text {Aeq, } \mathrm{T}}$, $\mathrm{L}_{\mathrm{Ceq}, \mathrm{T}}$, maximum, minimum and peak value $\mathrm{L}_{\text {Cpeak. }}$ As it can be noticed in figure 13, high values of the sound monitoring are observed during the AM-FDM process, where the $3 \mathrm{D}$ printer is working on creating the $3 \mathrm{D}$ piece presented in figure 3 .

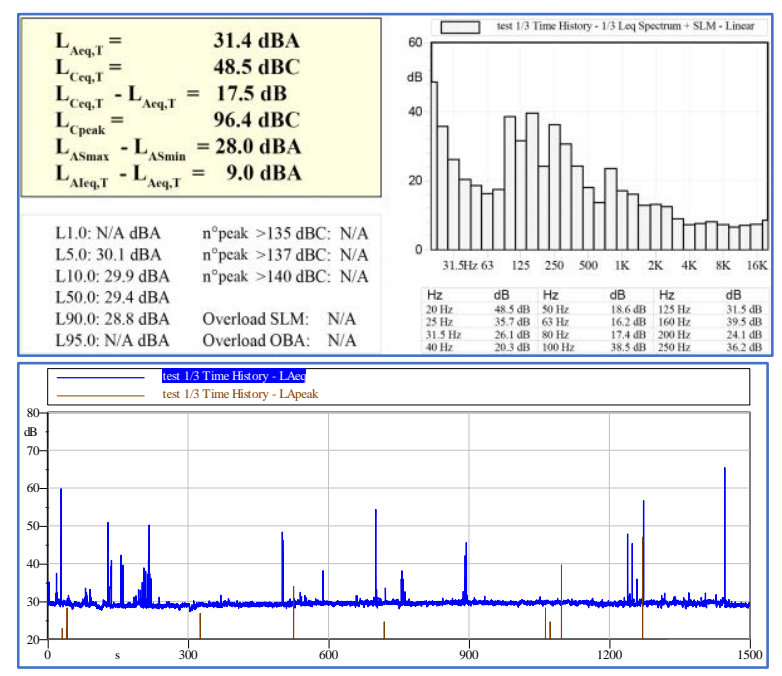

Fig. 13. Measured and logged sound signals using Larson Davis 831 sonometer

\subsection{Bayesian Networks Model for CBM}

Bayesian networks are graphical models consisting of nodes representing stochastic variables and directed links between the nodes, representing causal relationships. For a discrete model, each node has a discrete number of states, corresponding to the possible outcomes of the stochastic variable. If the stochastic variable is in fact continuous, it can be discretized so that each state represents an interval of values. 


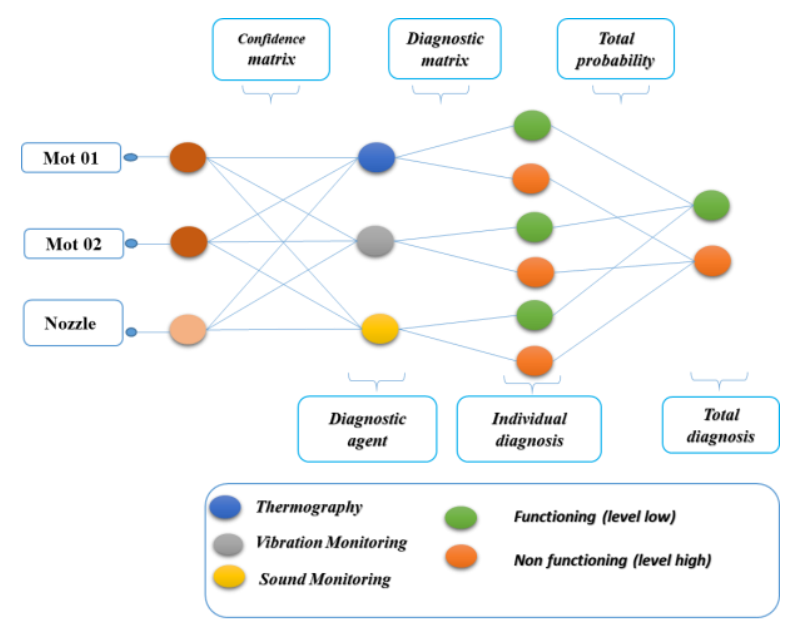

Fig. 14. Three-model Bayesian networks applied for achieving CBM

To define the Bayesian network, the conditional probability distribution must be specified for each node conditioned on parent nodes (the nodes pointing towards a node). For a node with no parents, the marginal distribution is specified. The network can then be used for calculating the probability of each state for each of the nodes, and these probabilities can be updated, when any of the nodes is observed [31], [32], [33], [34].

As shown in figure 12, it can be assumed that the faults of the 3D printer component (motors or nozzle) can be optimally detected by a hybrid of three diagnostic agents or fault detection methods. The probability of detection for each diagnostic agent is defined within a Confidence Matrix. Once the individual diagnosis of each agent is estimated and placed in a Diagnosis Matrix, the total probability theorem can be used to incorporate all probabilistic fault detection results into one final answer.

Bayesian network design with 3 inputs (M1 \& M2 \& Nozzle) is connected to 3 neurons or diagnostic agents (Thermography, Vibration Monitoring and Sound Monitoring). Each neuron is connected to individual diagnosis and all of them are combined as probabilities of Functioning or Non-functioning components. For example, if temperature of M1 has a high level, the 3D printer cannot function. And if the vibration of nozzle is very high, the $3 \mathrm{D}$ printer gives bad result, and the $3 \mathrm{D}$ piece is not printed properly.

\section{CONCLUSION}

The condition-based maintenance for the optimization of smart manufacturing process is absolutely critical for the proper functioning of the complex systems and devices. The paper addresses the condition-based maintenance (CBM) of the additive manufacturing (AM) processes, using three types of conditionmonitoring (CM): infrared thermography (IRT), vibration monitoring (VM) and sound monitoring (SM). Multiple software for different data acquisition, analysis and processing were studied and used:
ThermaCAM ${ }^{\circledR}$ Researcher ${ }^{\mathrm{TM}}$ from Flir for IRT, Noise \& Vibration Works (version 2.4.1) for noise and vibration analysis, VibSensor for vibration mobile recording, Cura and Simplify 3D for 3D printing. All the devices and equipment used were properly calibrated ensuring the experimental accuracy.

The measured data served to the development of a probabilistic model using Bayesian Networks, for a condition-based maintenance model in the case of the AM process. This technique and approach can represent a successful integration of a large number of data monitoring sets, with complex modelling and analysis capabilities, that can lead, in the end, at an optimisation of the AM process.

Summarizing, from the results obtained, it can be concluded that Bayesian probabilistic networks (BNs) can clearly contribute to the improvement of the CBM strategies, specifically when the data obtained from monitoring has non-linear behaviour and the classical mathematical models cannot be applied.

In conclusion, the paper addresses complex fundamental and experimental researches with wide application in Industrial Engineering field, and specifically in Condition-Based Maintenance. The proposed model, reproduced at larger scale, can be applied in industry, not only on CNC machines, as the 3D printer can be considered, but also to robotic assembly lines, 3D coordinate measurement systems, portal cranes, etc. The measurement of vibration, sound and temperature can be done continuously, and the monitored values can be integrated into a selfdiagnosis monitoring system, as those encountered to the modern cars, and, in an unfortunate event, when the machine receives a defective or abnormal data value, alarming systems are triggered and visuals appear and are signalled on specifically designed dashboards.

\section{ACKNOWLEDGEMENTS}

This work was supported by Erasmus+ Programme, KA2 - Capacity Building in Higher Education, project no. 586035-EPP-1-2017-1-DZ-EPPKA2-CBHE-JP, entitled Algerian National Laboratory for Maintenance Education - ANL Med, and was carried out in the framework of "Dunarea de Jos" University of Galati (UDJG), Romania, Faculty of Engineering, Department of Manufacturing Engineering, Laboratory of Mechatronics and Robotics in collaboration with Université M'Hamed Bougara de Boumerdes (UMBB), Faculty of Engineering Sciences, Department of Mechanical Engineering, Algeria.

\section{REFERENCES}

[1] Simeone A., Caggiano A., Boun L., Deng B., Intelligent cloud manufacturing platform for efficient resource sharing in smart manufacturing networks, Procedia CIRP, vol. 79, 2019, ISSN 22128271, pp. 233-238.

[2] Tao F., Qi Q., Liu A., Kusiak A., Data-driven smart manufacturing, Journal of Manufacturing Systems, vol. 48, part C, ISSN 0278-6125, 2018, pp. 157-169.

[3] Rastegari A., Bengtsson M., Implementation of Condition 
Based Maintenance in manufacturing industry - A pilot case study, 2014 International Conference on Prognostics and Health Management, pp. 1-8, 2014.

[4] Rastegari, A., Condition Based Maintenance in the Manufacturing Industry: From Strategy to Implementation, [Internet] [PhD dissertation]. Mälardalen University, 2017.

[5] Kumar A., Methods and Materials for Smart Manufacturing: Additive Manufacturing, Internet of Things, Flexible Sensors and Soft Robotics, Manufacturing Letters, vol. 15(B), ISSN 2213-8463, 2018, pp.122-125.

[6] *** ISO/ASTM 52900-12, Standard Terminology for Additive Manufacturing - General Principles - Terminology, 2015.

[7] Tofail S. A. M., Koumoulos E. P., Bandyopadhyay A., Bose.S., O'Donoghue L., Charitidis C., Additive manufacturing: scientific and technological challenges, market uptake and opportunities, Materials Today, vol. 21(1), ISSN 1369-7021, 2018, pp. 22-37.

[8] Solomon A., Rosenthal Y., D. Ashkenazi, Stern A., Structure AND Mechanical Behavior of Additive Manufactured Fused Deposition Modeling ABS, Annals of "Dunarea de Jos" University, of Galati, Fascicle XII, Welding Equipment and Technology, vol. 29, 2018, pp. 47-56.

[9] Kusiak A., Fundamentals of smart manufacturing: A multithread perspective, Annual Reviews in Control, vol. 47, ISSN 13675788, 2019, pp. 214-220.

[10] Alcácer V., Cruz-Machado V., Scanning the Industry 4.0: A Literature Review on Technologies for Manufacturing Systems, Engineering Science and Technology, an International Journal, vol. 22(3), ISSN 2215-0986, 2019, pp. 899-919.

[11]Zhong, R.Y., Xu X., Klotz E., Newman S. T., Intelligent Manufacturing in the Context of Industry 4.0: A Review, Engineering, vol. 3(5), ISSN 2095-8099, 2017, pp. 616-630.

[12] Jardine A. K. S., Lin D., Banjevic D., A review on machinery diagnostics and prognostics implementing condition-based maintenance, Mechanical Systems and Signal Processing, vol. 20(7), ISSN 0888-3270, 2006, pp. 1483-1510.

[13] Emami-Mehrgani B., Patrick Neumann W., Nadeau S., Bazrafshan M., Considering human error in optimizing production and corrective and preventive maintenance policies for manufacturing systems, Applied Mathematical Modelling, ISSN 0307-904X, vol. 40(3), 2016, pp. 2056-2074.

[14] Yepez P., Alsayyed B., Ahmad R., Intelligent assisted maintenance plan generation for corrective maintenance, Manufacturing Letters, vol. 21, ISSN 2213-8463, 2019, pp. 7-11.

[15] Fumagalli L., Macchi M., Giacomin A., Orchestration of preventive maintenance interventions, IFAC-PapersOnLine, vol. 50(1), ISSN 2405-8963,2017, pp. 13976-13981.

[16] Lee J., Bagheri B., Kao H. A., A Cyber-Physical Systems architecture for Industry 4.0-based manufacturing systems, Manufacturing Letters, Volume 3, 2015, pp. 18-23.
[17] Wan J., Tang S., Li D., Wang S., Liu C., Abbas H., Vasilakos A. V., A Manufacturing Big Data Solution for Active Preventive Maintenance, IEEE Transactions on Industrial Informatics, vol. 13(4), 2017, pp. 2039-2047.

[18] Yan J., Machinery prognostics and prognosis-oriented maintenance management, Singapore: Wiley, Internet resource, 2015.

[19] Belaid S., Rusu C. C., Smail A., Condition-Based Maintenance Methods for Smart Manufacturing Processes, 7th edition of the Scientific Conference of Doctoral Schools (SCDS-UDJG), 13th 14th of June 2019, Galati, Romania, poster.

[20]*** Creality 10S Pro 3D printer User manual,

https://www.creality3d.cn/creality3d-cr-10s-pro-p00253p1.html

[21]*** Creality 10S Pro 3D printer User manual, https://www.creality3d.cn/creality3d-cr-10s-pro-p00253p1.html [22]*** Nema 17 User manual,

http://www.logicbus.com/assets/PDF/27/Stepper\%20Motor\%20Ma nual.pdf

[23]*** Flir A20M user manual,

http://www.thermokameras.com/ir-

systeme/software/Handbuch_ResearchIR.pdf

[24]*** Vibration meter Quest model VI-100,

http://www.grupomeyer.com/imagenes/quest/vibraciones/100/vi100 .pdf

[25]*** Larson 831 Davis User Manual,

http://www.larsondavis.com

[26]*** Flirtools Software, https://www.flir.com/products/flir-

tools/

[27]*** Noise \& Vibration Works (NWWin)

http://www.ancoustics.com/index.php/software

[28]*** VibSensor user guide, http://www.now-

instruments.com/get-help/5-vibsensor-user-guide

[29]*** Cura software user manual,

https://ultimaker.com/software/ultimaker-cura

[30]*** Simplify 3D software manual,http://www.simplify3d.com/ [31] Jaramillo V. H., Ottewill J. R., Dudek R., Lepiarczyk D., Pawlik P., Condition monitoring of distributed systems using twostage Bayesian inference data fusion, Mechanical Systems and Signal Processing, vol. 87(A), ISSN 0888-3270, 2017, pp. 91-110, [32] Gao Y., Li B., Wang W., Xu W., Zhou C., Jin Z., Watching and Safeguarding Your 3D Printer: Online Process Monitoring Against Cyber-Physical Attacks, Proceedings of Proceedings of the ACM on Interactive, Mobile, Wearable and Ubiquitous Technologies, article 108, 2018, 27 pp.

[33] Efron B., Bayes' theorem in the 21st century, Science, vol. 340(6137), 2013, pp. 1177-1178.

[34] Cai B., Liu Y., Fan Q., Zhang Y., Liu Z., Yu S., Ji R., Multisource information fusion-based fault diagnosis of ground-source heat pump using Bayesian network, Applied Energy, vol. 114, 2014, pp. $1-9$. 